\title{
Scaling up alcohol intervention among youth and experiments of naturalistic settings
}

\author{
Eduardo J. Simoes
}

Published online: 29 January 2013

(c) Swiss School of Public Health 2013

The article "A quasi-randomized group trial of a brief alcohol intervention on risky single occasion drinking among secondary school students" (Gmel et al. 2012) contributes to the evidence around school-based interventions to prevent alcohol consumption among youngsters. Its divergent findings add to the mixed results common in systematic reviews around alcohol prevention.

Heavy alcohol drinking causes a host of chronic illnesses for the drinker, such as alcohol dependence, vascular disease (e.g., hypertension), hepatic cirrhosis, and various cancers, and contributes to accidental deaths. It also affects the well-being and health of people around the drinker. Alcohol is the world's third largest risk factor for disease burden, accounting for 4.9 million (4.5 million to 5.2 million) deaths and $5.5 \%(5.0-5.9)$ of global disability adjusted life-years in 2010 ( $\mathrm{Lim}$ et al. 2012). The toll of alcohol-related death among youth is also very high worldwide; 320,000 young people aged 15-29 years die annually, resulting in $9 \%$ of all deaths in that age group (WHO 2011). For people aged 15-49 years, alcohol is the leading risk factor worldwide followed by tobacco smoking (including second-hand smoke), high blood pressure, high body-mass index, diet low in fruits, drug use, and occupational risk factors for injuries (Lim et al. 2012). While WHO recommends a wide range of strategies and interventions to reduce alcohol impact on health, few target adolescents. We attribute this to lack of strong evidence for such interventions.

\section{E. J. Simoes $(\square)$}

Department of Health Management and Informatics, University of Missouri School of Medicine, CE707 CS\&E Bldg. DC006.00

One Hospital Drive, Columbia, MO 65212, USA

e-mail: simoese@health.missouri.edu

URL: http://www.hmi.missouri.edu/
In a summary of seven published Cochrane and nonCochrane Systematic reviews summarizing results from 430 papers from 1988 to 2005 on the prevention of alcohol use among youth, authors concluded that intervention effect was indeterminate or of limited effect (Baker et al. 2006). However, in a series of three recent Cochrane reviews on school, family, or multi-component prevention programs, some were shown to be effective in reducing alcohol misuse in youths (Larimer and Cronce 2002). Authors also concluded that results warranted a cautious interpretation, since bias and/or contextual factors could have affected trial results. That some studies show some effect and other studies show no effect for school-based alcohol prevention programs may be a reflection of the reality that school-based alcohol prevention programs are ineffective, with differences in results being attributed simply to variation of individual study (and sub-group analysis) effect size estimates around an actual zero effect, with some achieving statistical significance by chance (Ioannidis 2005).

In the study by Gmel et al., the effect of a brief alcohol intervention (BAI) based on motivational interviewing (MI) technique (Gmel et al. 2012, Miller and Rollnick 2002) was modified from its originally tested mode of individual-centered session to a group-level intervention. In addition, the original outcomes measures shown to be associated with individual-oriented $\mathrm{BAI}$ - use age of alcohol, initiation and average consumption of alcoholwere not used in the Gmel et al. study. Instead, Gmel et al. measured 'Risky single occasion drinking' (RSOD, also called binge drinking or alcohol use leading to intoxication). Also, unlike previous randomized designs that demonstrated the effectiveness of BAI delivered as individual-level sessions to prevent alcohol use among youth, Gmel et al. study is a quasi-experiment. 
It is possible that the lack of effect identified for BAI on RSOD by Gmel et al. is due to its group-oriented mode of delivery as opposed to an individual-level intervention. However, there is evidence that group-level intervention in the classroom is an effective strategy to prevent alcohol use in youth from elementary through high-school ages. A comprehensive Cochrane systematic review suggested that certain generic psychosocial and developmental prevention programs can be effective and could be considered as policy and practice options (Foxcroft and Tsertsvadze 2011). These include strategies for school settings such as the Life Skills Training Program, the Unplugged Program, and the Good Behavior Game.

Measuring an outcome such as RSOD as opposed to other alcohol-related outcomes could also have influenced negative results for a BAI effect on alcohol use among secondary school students. The impact of alcohol consumption on disease and injury is largely determined by two separate but related dimensions of drinking: total volume of alcohol consumed and pattern of drinking. There is evidence for youth-targeted programs to prevent health hazards associated with alcohol use such as automobilerelated accidents. A review of school-based intervention to reduce driving after drinking (DD) and riding with drinking driver (RDD) identified sufficient evidence for RDD and insufficient evidence for DD (Elder et al. 2005). Binge drinking or RSOD is more common among youth, and binge drinkers are 14 times more likely to report alcoholimpaired driving than non-binge drinkers (Naimi et al. 2003).

Gmel et al. article also underscores a needed discussion around evaluation of complex interventions, i.e., the necessary modifications of traditional study designs to address the realities of practice. Critics may view Gmel et al. study results as reflecting what they believe to be a failure of design to keep a high-level of internal validity. Thus, findings may be a result of biases that are unavoidable and uncontrolled. But for the evaluation of the group-level BAI effect on RSOD to be useful for policy making, Gmel et al. had to take special care to replicate usual and normal conditions of a school environment. They also made sure that adaptation of the intervention could be feasibly and acceptably implemented by instructors, mentors, and administrators, while being fully acceptable to students. In these circumstances, a randomized design can only be achieved by artificially recreating the environment and context where it takes place. Policy, planning, and management of such 'artificially-designed' intervention would probably lead to evaluation results that do not corroborate findings of 'gold standard' initial trials.

A broad range of alcohol consumption patterns, from occasional hazardous drinking to frequent binge drinking to daily heavy drinking, creates significant public health and safety problems in nearly all countries. Perhaps, it is time to scale up existing interventions that have been shown to be effective, regardless of the level of effectiveness. Perhaps, it is also the time to accept that we need to implement studies of necessary adaptations and replications of these interventions. Moreover, these studies should pay special attention to the evaluation of external validity and contextual factors that could support sustainability of effective programs.

\section{References}

Baker P, Young M, Thomas R (2006) A summary of published Cochrane and non-Cochrane Systematic reviews (from HealthEvidence.ca, The Community Guide and quality assessed systematic reviews (Database of Abstracts of Reviews of EffectsDARE): http://www.health.qld.gov.au/ph/documents/caphs/32104. pdf)

Elder RW, Nichols JL, Shults RA et al (2005) Effectiveness of school-based programs for reducing drinking and driving and riding with drinking drivers: a systematic review. Am J Prev Med 28(5S):288-304

Foxcroft DR, Tsertsvadze A (2011) Universal school-based prevention programs for alcohol misuse in young people. Cochrane Database of Syst Rev (5), Art No. CD009113. doi:10.1002/ 14651858.CD009113

Gmel G, Venzin V, Marmet K, Danko G, Labhart F (2012) Quasirandomized group trial of a brief alcohol intervention on risky single occasion drinking among secondary school students. Int J Public Health 57:935-944

Ioannidis JPA (2005) Why most published research findings are false. PLoS Med 2(8):e124. doi:10.1371/journal.pmed.0020124

Larimer ME, Cronce JM (2002) Identification, prevention and treatment: a review of individual-focused strategies to reduce problematic alcohol consumption by college students. J Stud Alcohol Suppl 14:148-163

Lim SS, Vos T, Flaxman AD et al (2012) A comparative risk assessment of burden of disease and injury attributable to 67 risk factors and risk factor clusters in 21 regions, 1990-2010: a systematic analysis for the Global Burden of Disease Study. Lancet 380:2224-2260

Miller WR, Rollnick S (2002) Motivational interviewing: preparing people for change, 2nd edn. Guilford Press, New York

Naimi TS, Brewer RD, Mokdad A, Denny C, Serdula MK, Marks JS (2003) Binge drinking among US adults. JAMA 289(1):70-75

WHO 2011. Global status report on alcohol and health: http://www. who.int/substance_abuse/publications/global_alcohol_report/en/ index.html 